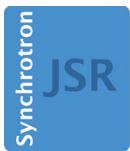

JOURNAL OF SYNCHROTRON RADIATION

ISSN 1600-5775

Received 11 September 2019

Accepted 15 December 2019

Edited by Y. Amemiya, University of Tokyo, Japan

Keywords: AP-XPS; SPEM; beamline; PAL (PLS-II).

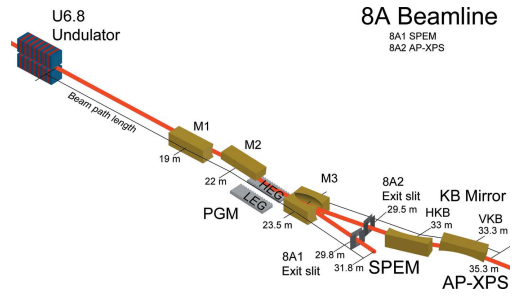

OPEN ๑ ACCESS

\section{AP-XPS beamline, a platform for operando science at Pohang Accelerator Laboratory}

\author{
Geonhwa Kim, ${ }^{a}$ Youngseok Yu, ${ }^{a}$ Hojoon Lim, ${ }^{a}$ Beomgyun Jeong, ${ }^{b}$ Jouhahn Lee, \\ Jaeyoon Baik, ${ }^{\mathrm{c} *}$ Bongjin Simon Mun ${ }^{\mathrm{a} *}$ and Ki-jeong Kim ${ }^{\mathrm{c} *}$ \\ ${ }^{a}$ Department of Physics and Photon Science, Gwangju Institute of Science and Technology, Gwangju 61005, \\ Republic of Korea, 'b Advanced Nano-Surface Research Group, Korea Basic Science Institute, Daejeon 34133, \\ Republic of Korea, and 'Beamline Research Division, Pohang Accelerator Laboratory, Pohang 37674, \\ Republic of Korea. *Correspondence e-mail: cla100@postech.ac.kr, bsmun@gist.ac.kr, kjkim@postech.ac.kr
}

Beamline 8A (BL 8A) is an undulator-based soft X-ray beamline at Pohang Accelerator Laboratory. This beamline is aimed at high-resolution ambientpressure X-ray photoelectron spectroscopy (AP-XPS), soft X-ray absorption spectroscopy (soft-XAS) and scanning photoemission microscopy (SPEM) experiments. BL 8A has two branches, 8A1 SPEM and 8A2 AP-XPS, that share a plane undulator, the first mirror (M1) and the monochromator. The photon beam is switched between the two branches by changing the refocusing mirrors after the monochromator. The acceptance angle of M1 is kept glancing at $1.2^{\circ}$, and $\mathrm{Pt}$ is coated onto the mirrors to achieve high reflectance, which ensures a wide photon energy range (100-2000 eV) with high resolution at a photon flux of $\sim 10^{13}$ photons $\mathrm{s}^{-1}$. In this article, the main properties and performance of the beamline are reported, together with selected experiments performed on the new beamline and experimental system.

\section{Introduction}

Currently, scientists and engineers are searching for possible solutions to mitigate serious imminent global issues such as climate change, deficit energy resource and air pollution. These issues seem to be independent, yet they are closely interconnected. Solutions can be found from various fields of science and engineering, for example environmental science, catalysis, nanotechnology and energy technology, and, in particular, material science can provide a starting platform for any possible solution.

In material science, identifying or modifying the 'surface/ interface' of the materials is the most important process as most physical or chemical reactions take places at the surface or interfaces (Ertl, 2015). While many analytical techniques are developed for studying the surface/interface, electron- or ion-based techniques provide highly surface sensitive information due to their short inelastic mean free path, e.g. ion scattering, secondary ion mass spectroscopy and X-ray photoemission spectroscopy. These tools have proven to be highly effective in studying the gas/solid surface (or interface) under ultrahigh vacuum (UHV) conditions. However, in the case of the liquid/solid surface (or interface) or the gas/solid interface under elevated pressure conditions, electron- or ionbased tools cannot be used.

In fact, since the early days of surface science, there has been a huge amount of effort in adapting UHV-based surfacescience techniques to ambient-pressure (AP) systems (Siegbahn, 1969; Somorjai, 1978; Joyner et al., 1979). Any surface chemical reaction mechanism occurring under UHV can differ 
significantly from that taking place under ambient-pressure conditions. As in situ surface studies have become increasingly important over the years, a number of innovative operando spectroscopy and microscopy techniques have also been developed (Shen, 1989; Hu et al., 1995; Hansen et al., 2001; Hendriksen \& Frenken, 2002; Donald, 2003; Hüfner, 2003; Ferrer et al., 2007; Forsberg et al., 2007). Ambient-pressure $\mathrm{X}$-ray photoemission spectroscopy (AP-XPS) is one of the prominent techniques that can provide surface/interface information under near ambient pressure conditions, i.e. in the $\sim$ mbar range (Siegbahn, 1969; Joyner et al., 1979; Ruppender et al., 1990; Ogletree et al., 2002; Salmeron \& Schlögl, 2008). AP-XPS, built with differential pumping electrostatic lens system schemes, has clearly made a big contribution to the operando science community (Bluhm, 2010).

X-ray photoemission spectroscopy (XPS), the predecessor of AP-XPS, has been demonstrated as an invaluable technique in the study of filled electronic states of solids, as well as helping to determine the nature of interactions between solid surfaces and molecular species. But there is one main barrier of the technique - that XPS measurements on clean surfaces for surface science are required to be performed under UHV conditions $\left(\sim 10^{-10} \mathrm{mbar}\right)$. This limitation was partially overcome with the development of differentially pumped AP-XPS systems, which began in the early 1970s (Siegbahn \& Siegbahn, 1973). Despite much effort, they suffered from poor electron yields due to the scattering of electrons in the gas phase. Then, with advances in electrostatic lens systems coupled with differential pumping of electron analyzer and high-flux synchrotron radiation sources, AP-XPS measurements became possible in the $100 \mathrm{mbar}$ pressure range. AP-XPS has been recognized by scientific communities as an important in situ tool to study water, environmental science, catalysis and many other important fields (Salmeron \& Schlögl, 2008; Starr et al., 2013; Shavorskiy et al., 2014). Since 2000, AP-XPS systems have been continuously installed at most major synchrotron radiation facilities around the world: ALS, BESSY, SSRL, MAX IV, SLS, ALBA, SOLEIL, SPring-8, Diamond, SSRF, and so on. Using these systems, many researchers have already published meaningful results in various fields (Artiglia et al., 2017; Timm et al., 2018; Kim et al., 2018; Yu et al., 2019; Soler et al., 2019; Diulus et al., 2019; Tesch et al., 2019; Cai et al., 2019).

Recently, beamline 8A (BL 8A) at Pohang Accelerator Laboratory (PAL) has been successfully reconstructed and an AP-XPS end-station is installed. Since 1999, BL 8A, the first undulator beamline at PAL, has been dedicated for surface/ interface and material science by providing scanning photoemission microscopy (SPEM) (8A1) and high-resolution photoemission spectroscopy (HR-PES) (8A2) (Shin et al., 1999). The undulator at BL 8A was designed to produce photon energies in the range $50-2000 \mathrm{eV}$, and BL $8 \mathrm{~A}$ has been one of the most popular beamlines at PAL as a user-friendly end-station that can provide both microscopic and spectroscopic techniques for surface/interface science. However, the practically available photon energy range at the end-station was gradually reduced to $100-800 \mathrm{eV}$ as beamline components including the optical system went out of exhaustive operation. User groups in surface/interface and material science have made continuous requests to recover the higher energy above $2000 \mathrm{eV}$ and introduce an additional advanced photoemission method, e.g. AP-XPS, for operando science. Finally, in December 2014, PAL and KBSI signed a Memorandum of Understanding for the construction of a new beamline and AP-XPS experimental system to initiate a joint research program in emerging fields of science.

In this paper, we outline the main properties and performance of the beamline as characterized during commissioning at the PAL storage ring (PLS II). For fertilizing new operando science, the beamline was designed with a wide photon energy range (100-2000 eV) with high photon resolution. A high photon flux, $\sim 10^{13}$ photon $\mathrm{s}^{-1}$, was achieved by keeping the M1 mirror acceptance angle glancing at $1.2^{\circ}$ as well as minimizing the number of total reflecting mirrors, four for the 8A1 SPEM branch and six for the 8A2 AP-XPS branch. We will also discuss the experimental capabilities and present a few showcase experiments which have been performed on the new beamline.

\section{Beamline overview}

\subsection{Photon source for BL $8 A$}

The U6.8 undulator for $\mathrm{BL} 8 \mathrm{~A}$ receives electron bunches of $3 \mathrm{GeV}$ and $400 \mathrm{~mA}$ from the storage ring of the PAL synchrotron (Shin et al., 2013; Hwang et al., 2014). The electron velocity is $0.9999999963733 c$ and the Lorentz factor $\gamma$, given as $\gamma=1 /\left(1-v^{2} / c^{2}\right)^{1 / 2}$, is 11742 . A total of 48 permanent magnets are displaced with a periodicity of $6.8 \mathrm{~cm}$ and the total length of the undulator is $3.3 \mathrm{~m}$. The mechanically allowed gap between the upper and lower magnet arrays is from 16 to $90 \mathrm{~mm}$, for which the deflection parameter $K$ of the undulator is from 5.769 to 0.105 , given by the following equation,

$$
K=\frac{e B_{0} \lambda_{\mathrm{u}}}{2 \pi m_{\mathrm{e}} c}
$$

where $B_{0}$ is the magnetic field at the centre of the undulator, $m_{\mathrm{e}}$ is the electron mass and $\lambda_{\mathrm{u}}$ is the period of the permanent magnets in the undulator. $B_{0}$ is a function of the gap and the geometry of the undulator.

With the given value of the parameter $K$, the photon energy $E$ of the $n$th harmonics, which is radiated with emission angle $\theta$ referred to the beam propagating direction, is determined by the following equation,

$$
E=\frac{2 n \gamma^{2} h c}{\lambda_{\mathrm{u}}\left[1+\left(K^{2} / 2\right)+\theta^{2} \gamma^{2}\right]} .
$$

As a result of equation (2), the U6.8 undulator covers the energy range from $71.236 \mathrm{eV}$ to $1248.94 \mathrm{eV}$ for the firstharmonic radiation. Including the third and fifth harmonics, the undulator can cover the entire energy range that the beamline was designed for, i.e. 100-2000 eV, with a flux density 


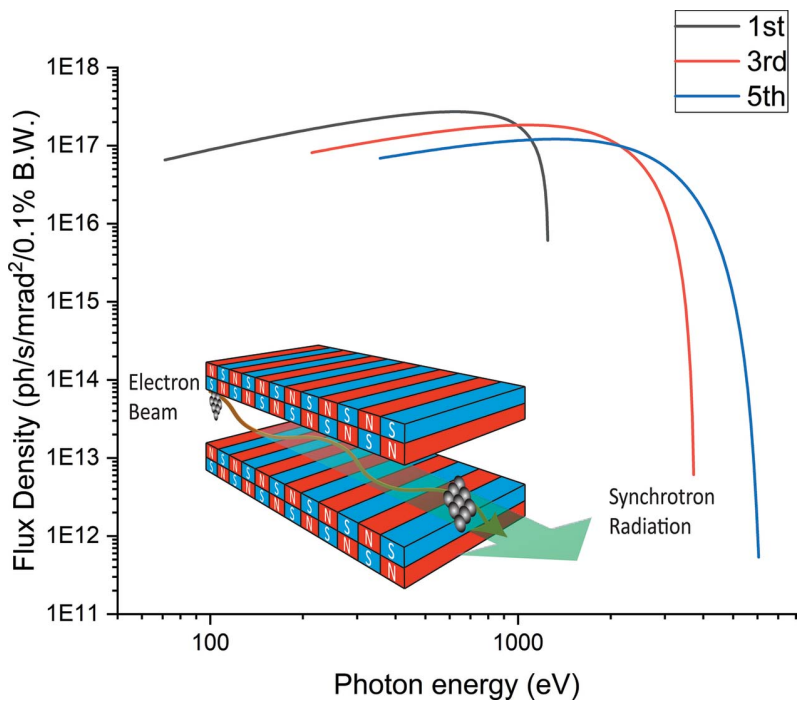

Figure 1

Calculated photon flux density of the U6.8 undulator radiation. The lines coloured black, red and blue represent the first, third and fifth harmonics, respectively. The inset shows a schematic diagram of the undulator.

of $10^{17}$ photons s${ }^{-1} \mathrm{mrad}^{-2}(0.1 \% \text { bandwidth })^{-1}$ theoretically as shown in Fig. 1. The radiated beam is linearly polarized.

\subsection{Beamline specifications}

The beamline optics are designed to accommodate the needs of the SPEM and AP-XPS end-stations, providing $\mathrm{X}$-rays over a wide energy range from 100 to $2000 \mathrm{eV}$. The undulator can operate in the energy range from $70 \mathrm{eV}$ up to $3000 \mathrm{eV}$, which corresponds to operation up to the third harmonic. The photon beam is collimated vertically by the first M1 mirror and delivered to the monochromator. The incident angle on the M1 mirror is $1.2^{\circ}$ to ensure high reflectance at higher energy $(\sim 1700 \mathrm{eV})$. The plane-grating monochromator (PGM) system is designed with SX-700-based optics to provide X-rays over a wide energy range (Petersen \& Baumgärtel, 1980). Fig. 2 shows the conceptual design of the PGM. The operation of the monochromator follows the grating equation described by $m \lambda / d=2 \cos \theta \sin (\theta+\beta)$, where $m$ is the diffraction order, $\lambda$ is the photon wavelength and $d$ is the line spacing corresponding to the ruling density of the

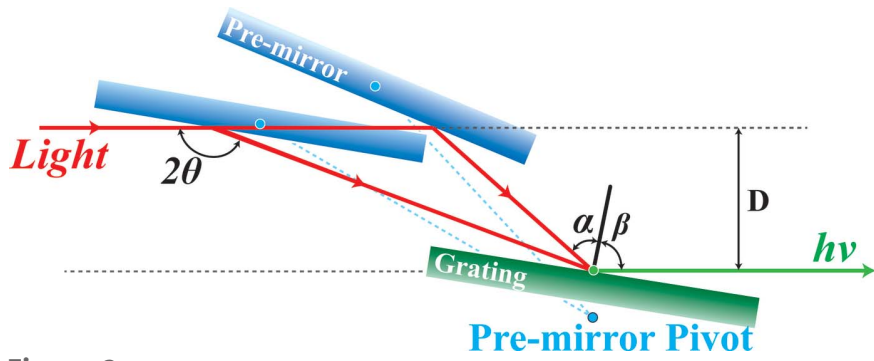

Figure 2

Schematic drawing of the operation mechanism of the PGM. The grating module rotates around the green pivotal point and the centre of the premirror rotates around the blue pivotal point close to the grating, which always transfers white light to the centre of the grating. grating. $D$, the vertical distance offset between the entering and exiting X-ray paths, is $15 \mathrm{~mm}$. By having a pre-collimated beam on the monochromator from the M1 mirror along the vertical direction, the fix constant, $C_{\mathrm{ff}}$, is allowed to vary freely. $C_{\mathrm{ff}}$ is given by $C_{\mathrm{ff}}=\cos \beta / \cos \alpha$, and $2 \theta$, the variable inclusive angle, is given by $2 \theta=\alpha-\beta$, where $\alpha>0, \beta<0$ and $2 \theta$ are shown in Fig. 2. This additional flexibility of $C_{\mathrm{ff}}$ enables the monochromator to be optimized for enhanced flux and improved energy resolution.

Two different gratings are installed in a single monochromator unit, where the low-energy grating (LEG) is for the photon energy range $100-1100 \mathrm{eV}$, and the high-energy grating (HEG) is for the photon energy range $900-2000 \mathrm{eV}$. The LEG is ruled with a 400 lines $\mathrm{mm}^{-1}$ line density and $16 \mathrm{~nm}$ groove depth, and HEG is ruled with a 500 lines $\mathrm{mm}^{-1}$ line density and $8 \mathrm{~nm}$ groove depth.

The diffracted beam from the monochromator is focused onto the exit slit and switched by inserting the M3-1 (SPEM, 8A1) or M3-2 (AP-XPS, 8A2) toroidal mirror. As a result, the monochromatic beam after the M3 mirrors focuses onto the exit slit with a beam size of $210 \mu \mathrm{m}$ (horizontal, $\mathrm{H}) \times 5.9 \mu \mathrm{m}$ (vertical, V) for $8 \mathrm{~A} 1$ and $280 \mu \mathrm{m}(\mathrm{H}) \times 7.1 \mu \mathrm{m} \mathrm{(V)}$ for $8 \mathrm{~A} 2$. The aperture size of the exit slit can be determined by controlling four-way blades $(x-y)$. Finally it is designed to achieve an energy resolving power of more than 5000 in the energy range that each grating can provide, when the vertical aperture size is kept at $40 \mu \mathrm{m}$, and LEG and HEG are operated with $C_{\mathrm{ff}}=2.5$ and $C_{\mathrm{ff}}=1.7$, respectively, with negative diffraction order.

In the SPEM (8A1) beamline, this monochromatic beam passes through a Fresnel zone plate and reaches the sample with a beam size of $100 \mathrm{~nm}$. In the AP-XPS (8A2) beamline, Kirkpatrick-Baez (KB) mirrors, located at the end of the photon transfer line, tightly refocus the monochromatic beam onto the AP-XPS sample position. The FWHM of the beam size at the focal spot of the AP-XPS sample is less than $50 \mu \mathrm{m}$ $(\mathrm{V}) \times 50 \mu \mathrm{m}(\mathrm{H})$. The beamline's optical layout and design are schematically shown in Fig. 3. The optical and geometrical specifications of each optical element are listed in Table 1.

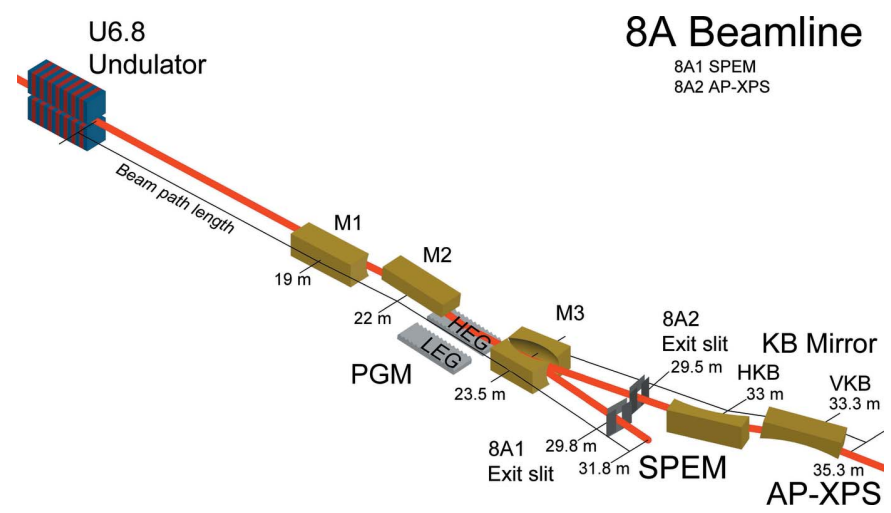

Figure 3

Optical layout and design of BL 8A. From the undulator, M1 horizontal focusing mirror, monochromator system with M2 and gratings, M3 and M3' branching mirror for SPEM and AP-XPS, respectively, and exit slits and KB mirror system for AP-XPS are located. 
Table 1

Beamline optics specifications.

\begin{tabular}{|c|c|c|c|c|c|c|}
\hline Name of optics & M1 mirror & M2 pre-mirror & Gratings & $\begin{array}{c}\text { M3-1 (8A1) / } \\
\text { M3-2 (8A2) }\end{array}$ & HKB (8A2) & VKB (8A2) \\
\hline Position from source & $19 \mathrm{~m}$ & $22 \mathrm{~m}$ & $23.5 \mathrm{~m}$ & $22 \mathrm{~m} / 25 \mathrm{~m}$ & $19 \mathrm{~m}$ & $19 \mathrm{~m}$ \\
\hline Shape & Cylindrical & Plane & $\begin{array}{l}\text { Plane } \\
\text { LEG: } 4001 \mathrm{~mm}^{-1} \\
\text { HEG: } 5001 \mathrm{~mm}^{-1}\end{array}$ & Toroidal & Cylindrical & Cylindrical \\
\hline Function & Vertical collimation & Just reflection & Diffraction & Focusing to exit slit & Horizontal refocusing & Vertical refocusing \\
\hline Tangential radius & Infinite & Infinite & Infinite & $32000 \mathrm{~cm} / 40000 \mathrm{~cm}$ & $10000 \mathrm{~cm}$ & $7550 \mathrm{~cm}$ \\
\hline Sagittal radius & $8012.3 \mathrm{~mm}$ & Infinite & Infinite & $4400 \mathrm{~mm} / 3138 \mathrm{~mm}$ & Infinite & Infinite \\
\hline Substrate material & $\begin{array}{l}\text { Glidcop (internal } \\
\text { cooling) }\end{array}$ & $\begin{array}{l}\text { Glidcop (internal } \\
\text { cooling) }\end{array}$ & $\begin{array}{l}\text { Single-crystal } \\
\text { silicon }\end{array}$ & $\begin{array}{l}\text { Single-crystal } \\
\text { silicon }\end{array}$ & $\begin{array}{l}\text { Single-crystal } \\
\text { silicon }\end{array}$ & $\begin{array}{l}\text { Single-crystal } \\
\text { silicon }\end{array}$ \\
\hline Incident angle & $1.2^{\circ}$ (in and out) & $\begin{array}{l}\text { Variable } \\
\qquad\left(0.9^{\circ}-5.0^{\circ}\right)\end{array}$ & $\begin{array}{l}\text { Variable } \\
\qquad\left(0.9^{\circ}-5.0^{\circ}\right)\end{array}$ & $2.0^{\circ} / 1.5^{\circ}$ & $1.5^{\circ}$ (in and out) & $2.0^{\circ}$ (in and out) \\
\hline Coating thickness $(\AA)$ & $\mathrm{Au}(600 \AA)$ & $\mathrm{Au}(600 \AA)$ & $\mathrm{Au}(600 \AA)$ & $\mathrm{Au}(600 \AA)$ & $\mathrm{Au}(600 \AA)$ & $\mathrm{Au}(600 \AA)$ \\
\hline $\begin{array}{l}\text { Beam size @830 eV } \\
(\mu \mathrm{m})\end{array}$ & $1240 \times 610$ & $1330 \times 610$ & $1360 \times 610$ & $\begin{array}{c}1370 \times 4060 / \\
1370 \times 4060\end{array}$ & $760 \times 2370$ & $570 \times 2900$ \\
\hline $\begin{array}{l}\text { Footprint @ } 830 \text { eV } \\
\quad(\mu \mathrm{m})\end{array}$ & $58400 \times 606$ & $1330 \times 17600$ & $1360 \times 119900$ & $\begin{array}{l}49500 \times 4060 / \\
54600 \times 4060\end{array}$ & $29100 \times 2370$ & $570 \times 78700$ \\
\hline
\end{tabular}

\section{Beamline performance: photon resolution and} photon flux

Fig. 4 shows the nitrogen $K$-edge absorption spectrum of gasphase $\mathrm{N}_{2}$, which indicates the resolving power of the grating. The measurement was taken using a gas cell located between the exit slit and the KB mirrors of beamline $8 \mathrm{~A} 2$ with an exit slit opening of $40 \mu \mathrm{m}(\mathrm{V}) \times 200 \mu \mathrm{m}(\mathrm{H})$. The gas cell was composed of two parallel plates and filled with $\mathrm{N}_{2}$ gas at a pressure of $10^{-5}$ mbar. One of the plates is biased by $+100 \mathrm{~V}$ and pushes photo-ionized $\mathrm{N}_{2}$ molecules, and the other detects the collision of ions to itself by measuring current outputs.

The spectrum was deconvoluted with a Voigt function for which the Lorentzian and Gaussian widths were $80 \mathrm{meV}$ and $40 \mathrm{meV}$, respectively. Since the Lorentzian width originates from lifetime broadening, the smaller value for this beamline compared with those of other soft X-ray beamlines (Lee \& Shin, 2001; Watanabe et al., 2004) can be due to using lower $\mathrm{N}_{2}$ gas pressure and providing fewer collision opportunities

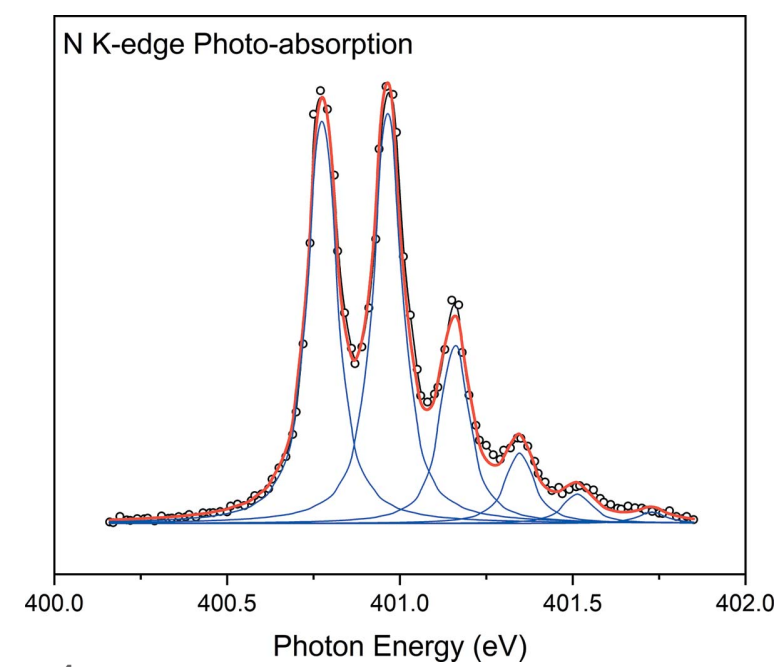

Figure 4

Photo-absorption spectrum of the nitrogen $K$-edge taken under $10^{-5}$ mbar $\mathrm{N}_{2}$ gas pressure. The best deconvolution using a Voight function allows an $80 \mathrm{meV}$ Lorentzian width and $40 \mathrm{meV}$ Gaussian width. between ions and electrons. Additionally, the low signal-tonoise ratio of these spectra may come from the low $\mathrm{N}_{2}$ gas pressure. At $400 \mathrm{eV}$ photon energy, the resolving power $E / \Delta E$ is estimated to be $400 \mathrm{eV} / 40 \mathrm{meV}=10000$, which matches precisely the designed value of the LEG.

Also, the photon flux at the end-station, which will be used as an important value in actual experiments, was measured using a silicon photodiode (IRD 100G) located right after the exit slit for the 8A1 SPEM end-station (Gullikson et al., 1996). In the most frequently used energy range of $100-2000 \mathrm{eV}$ in both beamlines, the photon flux could be estimated as $\sim 10^{13}$ photons s $^{-1}$.

\section{Experimental station}

4.1. Windowless aperture system with differential pumping stages: buffering the pressure gap between the beamline and the experimental chamber

The main obstacle in performing AP-XPS experiments is due to the elevated pressure in the main chamber in which experiments are conducted, which conflicts with the UHV requirement of the beamline. In many cases, a photon-transparent window, e.g. silicon nitride $\left(\mathrm{Si}_{3} \mathrm{~N}_{4}\right)$ or aluminium membrane (Al, thickness of $\sim 100 \mathrm{~nm}$ ), has been installed between the beamline and AP-XPS experiment chamber to maintain the UHV condition of the beamline. BL 8A2, however, adopts a windowless differential pumping station (SPECS, Germany), installed between the experimental system and beamline with an aperture system with $200 \mu \mathrm{m}$ diameter capillary. Using four pumping stages and four apertures (with diameters of $6 \mathrm{~mm}, 5 \mathrm{~mm}, 4 \mathrm{~mm}$ and $3 \mathrm{~mm}$ in order from upstream), the pressure at the KB-mirror chamber can be maintained at $\sim 10^{-9}$ mbar.

\subsection{Experimental components in AP-XPS}

The 8A2 AP-XPS end-station is aimed at surface studies under near ambient pressure conditions (up to 25 mbar, a pressure where liquid-phase water can exist at room 


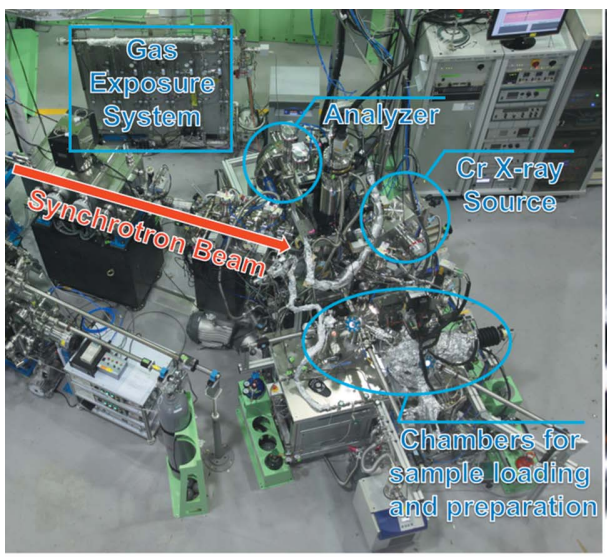

(a)

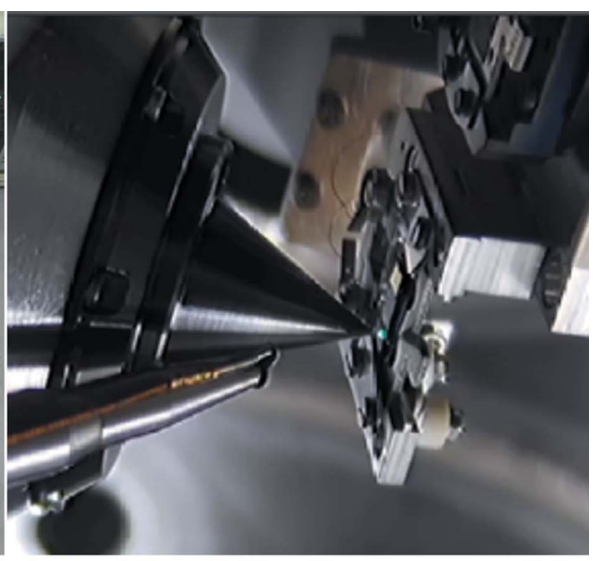

(b)

Figure 5

(a) Main experimental components of the AP-XPS end-station. (b) Beam spot site inside the analysis chamber. The angle between the analyser cone and beamline is $55^{\circ}$ and the angle between the analyser cone and the $\mathrm{Cr} \mathrm{X}$-ray source is $79^{\circ}$.

temperature) as well as UHV conditions. The end-station consists of preparation and analysis chambers with two manipulators: one for preparing a well defined sample surface in UHV conditions and the other for AP-XPS measurements, as can be seen in Fig. 5(a). The preparation chamber includes the usual equipment for cleaning and annealing surfaces $\left(\mathrm{Ar}^{+}\right.$ ion sputter gun and e-beam heater) and characterization of surface structure (LEED optics). There is a load-lock chamber for transferring the sample into the UHV chamber without venting the whole system. The analysis chamber, which has a backfilling configuration, is equipped with a SPECS Phoibos NAP 150 hemispherical analyzer for XPS. A Cr K $\alpha$ X-ray source $(h v=5.417 \mathrm{keV})$ is also installed in the main chamber as an auxiliary photon source, and allows the bulk property to be analyzed with a higher probing depth by detecting photoelectrons with high kinetic energy. On the sample manipulator in the analysis chamber there are three sample stages for cooling down the sample using liquid nitrogen, annealing the sample using an IR laser (Ostec, up to $1500 \mathrm{~K}$ ) and direct-

Table 2

Brief information for the AP-XPS experimental system.

\begin{tabular}{|c|c|}
\hline Electron analyzer & $\begin{array}{l}\text { SPECS Phoibos NAP } 150 \text { hemispherical } \\
\text { analyser with 2D delay line detector }\end{array}$ \\
\hline Photon energy & 100-2000 eV, 5417 eV (Cr X-ray source) \\
\hline Pressure & $\mathrm{UHV} \sim 25$ mbar \\
\hline \multirow[t]{3}{*}{ Temperature control } & Liquid nitrogen $(150 \mathrm{~K})$ \\
\hline & IR laser heater (up to $1500 \mathrm{~K}$ ) \\
\hline & Direct-current heating (up to $1500 \mathrm{~K}$ ) \\
\hline Gases & $\mathrm{N}_{2}, \mathrm{O}_{2}, \mathrm{CO}_{2}, \mathrm{CO}, \mathrm{Ar}, \mathrm{Ne} \ldots$ \\
\hline \multirow[t]{4}{*}{ Equipment } & Low-energy electron diffraction (LEED) \\
\hline & Ion gun \\
\hline & Electron beam heater \\
\hline & Residual gas analyser (RGA) \\
\hline \multirow[t]{6}{*}{ Applicable research area } & Physics at surface and interface \\
\hline & Gas-phase heterogeneous catalysis \\
\hline & $\begin{array}{l}\text { Electrochemistry (battery, fuel cell, corrosion, } \\
\text { electrocatalysis) }\end{array}$ \\
\hline & Photovoltaic \\
\hline & Environmental science \\
\hline & Biological systems ... \\
\hline
\end{tabular}

current heating of semiconductor samples, respectively. Fig. 5(b) shows the sample configuration inside the sample analysis chamber. Brief information on the AP-XPS experimental system is summarized in Table 2 .

\subsection{Probing depth change of $\mathrm{SiO}_{2} / \mathrm{Si}$ on photon energy}

To test the beamline photon energy and the analyzer, XPS measurements were carried out on a $\mathrm{SiO}_{2} / \mathrm{Si}$ (100) sample for various photon energies. Fig. 6 shows the Si $2 p$ XPS spectral changes for various photon energies under UHV conditions. For ease of comparison and avoidance of the difference by photoionization cross section, the spectra are normalized by the maximum intensity of the bulk Si $2 p_{3 / 2}$ peak. Since the natural oxide was already formed on the surface, the oxide peak of $\mathrm{Si} 2 p$, whose intensity varies with photon energy, follows the typical escape depth curve of an electron. Using a photon energy of $150 \mathrm{eV}$ to $1100 \mathrm{eV}$ for the LEG, all the data points were collected only once with a $0.1 \mathrm{~s}$ dwell time and $20 \mathrm{meV}$ step size at the most optimized position. The pass energy was $2 \mathrm{eV}$ from $150 \mathrm{eV}$ to $700 \mathrm{eV}$ and $10 \mathrm{eV}$ from $800 \mathrm{eV}$ to $1100 \mathrm{eV}$. When the Si $2 p$ core-level was measured using photon energies of $1586 \mathrm{eV}$ and $1950 \mathrm{eV}$, for the HEG, the accumulation number is increased to overcome the reduced photon flux by five times and ten times, respectively, with $0.1 \mathrm{~s}$ dwell time and $50 \mathrm{eV}$ pass energy. In summary, we would like to demonstrate the practical photon energy range by measuring the $\mathrm{Si} 2 p$ core-level on $\mathrm{SiO}_{2} / \mathrm{Si}(100)$ and prove that the available photon energy range is $100-2000 \mathrm{eV}$ at $\mathrm{BL} 8 \mathrm{~A}$.

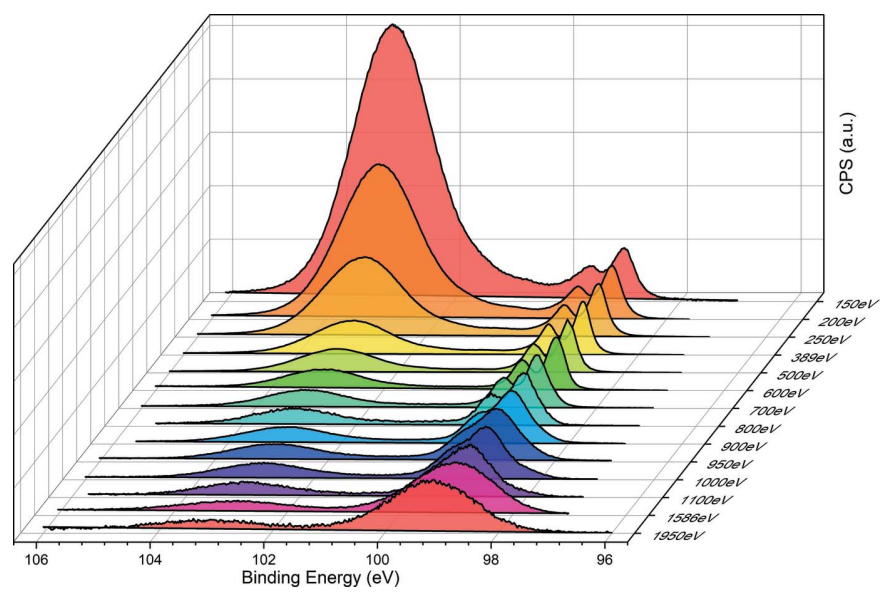

Figure 6

Si $2 p$ spectra corresponding to photon energies from $150 \mathrm{eV}$ to $1950 \mathrm{eV}$. The spectra are normalized with respect to the intensity of the $\mathrm{Si} 2 p_{3 / 2}$ peak. For the LEG, the energy region from $150 \mathrm{eV}$ to $1100 \mathrm{eV}$ is covered, while the HEG covered $1586 \mathrm{eV}$ to $1950 \mathrm{eV}$. 
(a)

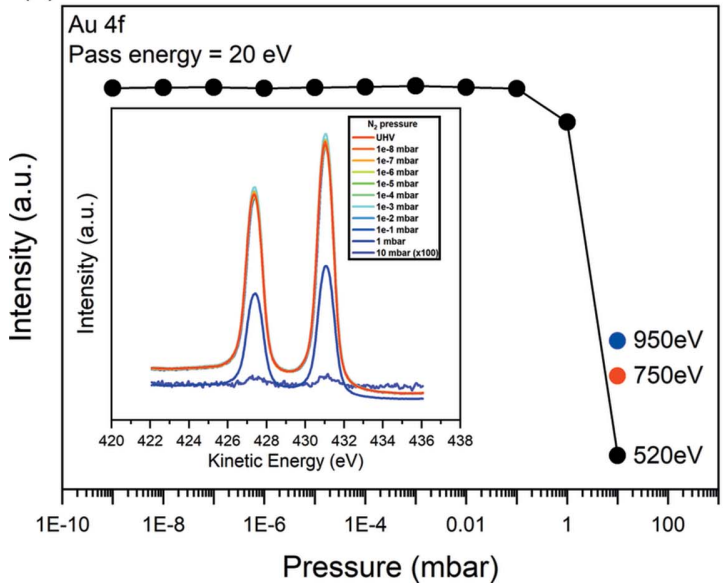

(b)

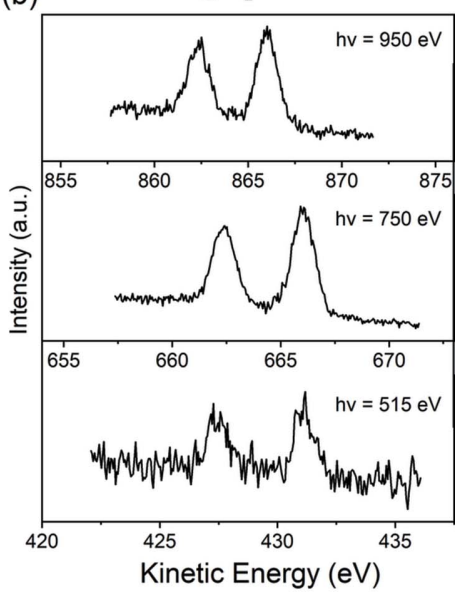

Figure 7

(a) Au $4 f$ intensity change at elevated $\mathrm{N}_{2}$ pressure. The black dots show the maximum intensities of $\mathrm{Au} 4 f_{7 / 2}$ peaks acquired from the spectra in the inset of $(a)$. (b) Raw spectra of Au $4 f$ taken under a 10 mbar $\mathrm{N}_{2}$ gas environment. All the spectra are collected in one scan.

\subsection{Intensity change of $A u 4 f_{7 / 2}$ under $N_{2}$ environments}

A simple performance test of AP-XPS was carried out with an $\mathrm{Au}$ reference. Fig. 7(a) shows the intensity change of $\mathrm{Au} 4 f_{7 / 2}$ for a gradual change of the $\mathrm{N}_{2}$ pressure at a photon energy of $520 \mathrm{eV}$. All of the spectra in Fig. 7 are acquired with a $0.1 \mathrm{~s}$ dwell time, $50 \mathrm{eV}$ pass energy, $50 \mathrm{meV}$ step energy, $3 \mathrm{~mm} \times 25 \mathrm{~mm}$ analyzer slit and $40 \mu \mathrm{m}(\mathrm{V}) \times 200 \mu \mathrm{m}(\mathrm{H}) 8 \mathrm{~A} 2$ exit slit. All signals are normalized using the beam current at the $\mathrm{KB}$ mirror. As the pressure of $\mathrm{N}_{2}$ is varied from $10^{-9}$ to 0.1 mbar, $\mathrm{Au} 4 f_{7 / 2}$ has almost the same intensity. At 1 mbar, the signal intensity starts to decrease and reduces rapidly in the 1-10 mbar range. At 10 mbar, the signal is very low due to the short inelastic mean free path of the photoelectrons which cannot overcome the distance between the sample and the nozzle of the spectrometer. In practice, signal is collected sufficiently to distinguish components in the spectrum up to 7-8 mbar in the case of $\mathrm{N}_{2}$ gas for photon energies of the LEG. Fig. 7(b) shows the $\mathrm{Au} 4 f_{7 / 2}$ spectra at photon energies of $750 \mathrm{eV}$ and $950 \mathrm{eV}$ at 10 mbar. It shows an increased intensity because of the prolonged inelastic mean free path of the electrons at higher photon energy.

4.5. Photo-induced nitrogen doping on graphene/Ge at near ambient pressure

An investigation of the photoinduced effect during the $\mathrm{N}_{2}$ gas pressurization onto graphene/Ge is a good example of AP-XPS. The graphene on (a) Before Pressurization

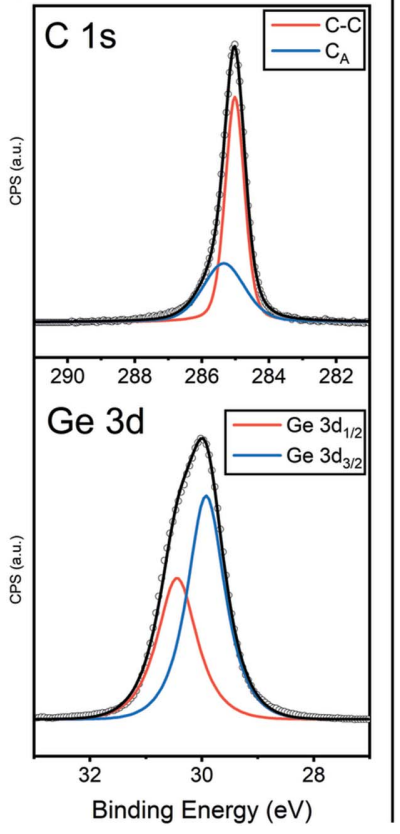

(b) After Pressurization

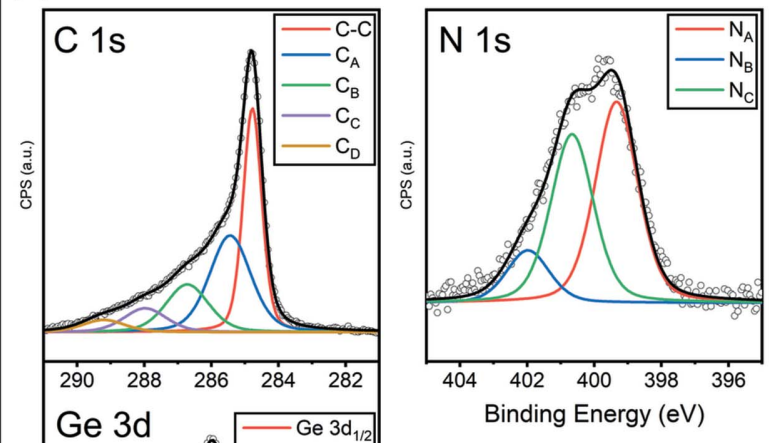

During the whole experiment, no heat or other gases are applied on the samples. Fig. 8 shows the fitting results of $\mathrm{C} 1 \mathrm{~s}$, Ge $3 d$ and $\mathrm{N} 1 s$ spectra of the exposed sample measured at UHV before and after pressurization, and the LEED patterns of the exposed and unexposed samples.

As shown in Fig. 8(b), several components of $\mathrm{N} 1 s$ at binding energy $399.3 \mathrm{eV}\left(\mathrm{N}_{\mathrm{A}}\right), 400.7 \mathrm{eV}\left(\mathrm{N}_{\mathrm{B}}\right)$ and $402 \mathrm{eV}\left(\mathrm{N}_{\mathrm{C}}\right)$ were found after the pressurization only for the photon-irradiated sample and only for the irradiated spot. These components are not reduced or removed even by annealing or

Ge (110) substrate system has been Yang et al., 2019), but many of its properties are not yet characterized. graphene/Ge samples are prepared as previously reported (Yang et al., 2019), and each is located at different stages in the main chamber manipulator to tion by exposing only one of them to a synchrotron beam $(h v=700 \mathrm{eV})$ during pressurization. $\mathrm{N}_{2}$ gas pressure was trace the generation of nitrogen species on the surface of the sample, and then pumped out to UHV again. By that process, the same atmospheric conditions are applied to both samples. 
ageing in UHV. Simultaneously, C $1 s$ of final states has an enhanced shoulder in the higher binding energy region compared with its initial state, shown in Fig. 8(a). On the other hand, Ge $3 d$ shows no change during the process. Additionally, beam exposure did not affect the LEED pattern of graphene as shown in Fig. 8(c). Therefore, it can be inferred that nitrogen atoms have some interaction with carbon atoms by irradiation but do not destroy the overall 2D structure of graphene. In previous investigations (Shao et al., 2010; Usachov et al., 2011; Mokhtar Mohamed et al., 2018; Xu et al., 2018), mainly three different types of nitrogen bonding to carbon atoms are reported in the N-doped graphene: pyridinic, pyrrolic and graphitic bonding. Based on this, the enhanced shoulder of the $\mathrm{C} 1 \mathrm{~s}$ peak could be deconvoluted into three peaks at binding energy $286.7 \mathrm{eV}\left(\mathrm{C}_{\mathrm{B}}\right), 288.0 \mathrm{eV}$ $\left(\mathrm{C}_{\mathrm{C}}\right)$ and $289.2 \mathrm{eV}\left(\mathrm{C}_{\mathrm{D}}\right)$, and they might be coupled with $\mathrm{N}_{\mathrm{A}}$, $\mathrm{N}_{\mathrm{B}}$ and $\mathrm{N}_{\mathrm{C}}$, respectively.

Since only the exposed sample has nitrogen-related species during the pressurization, those components can be thought of as being produced by the photo-induced effect. This result is quite encouraging because it can be a new method to synthesize $\mathrm{N}$-doped graphene. Further research will be conducted for more detailed information.

\section{Summary}

The AP-XPS beamline offers a platform for operando experiments at UHV and ambient-pressure conditions. With the combination of a soft X-ray $(100-2000 \mathrm{eV})$ and Cr X-ray source, two photon sources allows the electronic structure of matter to be studied from UHV to elevated pressures up to 25 mbar both at the surface and in the bulk. Beamline performance tests demonstrated that the beamline meets the design parameters very well. The estimated photon resolving power through the $\mathrm{N} K$-edge is more than 10000 , and the photon flux measured using an IRD photodiode is $\sim 10^{13}$ photon $^{-1}$. AP-XPS measurements of the Si and $\mathrm{Au}$ sample under elevated pressure also meet the expected performance while AP-XPS spectra of the graphene/Ge system under nitrogen pressure demonstrated the presence of the photo-induced effect. The beamline and AP-XPS endstation are currently open to public users at the $3.0 \mathrm{GeV}$ ring at PAL.

\section{Funding information}

Financial support was provided by the National Research Foundation of Korea (2015R1A5A1009962, NRF2019R1A2C2008052) and the GIST Research Institute Grant funded by the Gwangju Institute of Science and Technology 2019. Experiments at PLS-II were supported in part by MSICT and POSTECH, and NRF-2018R1D1A1B07048177, NRF-2016R1D1A1B03936348, NRF-2019M3D1A1079309. Building of the APXPS end-station was funded by MSICTKBSI-C39119.

\section{References}

Artiglia, L., Orlando, F., Roy, K., Kopelent, R., Safonova, O., Nachtegaal, M., Huthwelker, T. \& van Bokhoven, J. A. (2017). J. Phys. Chem. Lett. 8, 102-108.

Bluhm, H. (2010). J. Electron Spectrosc. Relat. Phenom. 177, 71-84. Cai, J., Dong, Q., Han, Y., Mao, B. H., Zhang, H., Karlsson, P. G., Åhlund, J., Tai, R. Z., Yu, Y. \& Liu, Z. (2019). Nucl. Sci. Tech. 30, 81.

Diulus, J. T., Frederick, R. T., Li, M., Hutchison, D. C., Olsen, M. R., Lyubinetsky, I., Árnadóttir, L., Garfunkel, E. L., Nyman, M., Ogasawara, H. \& Herman, G. S. (2019). ACS Appl. Mater. Interfaces, 11, 2526-2534.

Donald, A. M. (2003). Nat. Mater. 2, 511-516.

Ertl, G. (2015). Nobel Lecture: Reactions at Surfaces: From Atoms to Complexity, doi: 10.1002/anie.200800480.

Ferrer, S., Ackermann, M. D. \& Lundgren, E. (2007). MRS Bull. 32, 1010-1014.

Forsberg, J., Duda, L. C., Olsson, A., Schmitt, T., Andersson, J., Nordgren, J., Hedberg, J., Leygraf, C., Aastrup, T., Wallinder, D. \& Guo, J. H. (2007). Rev. Sci. Instrum. 78, 083110.

Gullikson, E. M., Korde, R., Canfield, L. R. \& Vest, R. E. (1996). J. Electron Spectrosc. Relat. Phenom. 80, 313-316.

Hansen, T. W., Wagner, J. B., Hansen, P. L., Dahl, S., Topsøe, H. \& Jacobsen, C. J. H. (2001). Science, 294, 1508-1510.

Hendriksen, B. L. M. \& Frenken, J. W. M. (2002). Phys. Rev. Lett. 89, 046101.

Hu, J., Xiao, X. D., Ogletree, D. F. \& Salmeron, M. (1995). Science, 268, 267-269.

Hüfner, S. (2003). Photoelectron Spectroscopy: Principles and Applications, 3rd rev. ed. Berlin, Heidelberg: Springer.

Hwang, I., Huang, J. Y., Kim, M., Lee, B. J., Kim, C., Choi, J. Y., Kim, M. H., Lee, H. S., Moon, D., Lee, E. H., Kim, D. E., Nam, S. H., Shin, S. \& Cho, M. (2014). Rev. Sci. Instrum. 85, 055113.

Joyner, R. W., Roberts, M. W. \& Yates, K. (1979). Surf. Sci. 87, 501509.

Kim, J., Park, W. H., Doh, W. H., Lee, S. W., Noh, M. C., Gallet, J. J., Bournel, F., Kondoh, H., Mase, K., Jung, Y., Mun, B. S. \& Park, J. Y. (2018). Sci. Adv. 4, eaat3151.

Kim, K. J., Yang, S., Park, Y., Lee, M., Kim, B. \& Lee, H. (2013). J. Phys. Chem. C, 117, 2129-2134.

Lee, M. K. \& Shin, H. J. (2001). Nucl. Instrum. Methods Phys. Res. A, 467-468, 508-511.

Mokhtar Mohamed, M., Mousa, M. A., Khairy, M. \& Am, A. A. (2018). ACS Omega, 3, 1801-1814.

Ogletree, D. F., Bluhm, H., Lebedev, G., Fadley, C. S., Hussain, Z. \& Salmeron, M. (2002). Rev. Sci. Instrum. 73, 3872-3877.

Petersen, H. \& Baumgärtel, H. (1980). Nucl. Instrum. Methods, 172, 191-193.

Ruppender, H. J., Grunze, M., Kong, C. W. \& Wilmers, M. (1990). Surf. Interface Anal. 15, 245-253.

Salmeron, M. \& Schlögl, R. (2008). Surf. Sci. Rep. 63, 169-199.

Shao, Y., Zhang, S., Engelhard, M. H., Li, G., Shao, G., Wang, Y., Liu, J., Aksay, I. A. \& Lin, Y. (2010). J. Mater. Chem. 20, 7491-7496.

Shavorskiy, A., Karslioglu, O., Zegkinoglou, I. \& Bluhm, H. (2014). Synchrotron Radiat. News, 27(2), 14-23.

Shen, Y. R. (1989). Nature, 337, 519-525.

Shin, H.-J., Chung, Y. \& Kim, B. (1999). J. Electron Spectrosc. Relat. Phenom. 101-103, 985-989.

Shin, S., Kwon, S., Kim, D. T., Kim, D. E., Kim, M., Kim, S. H., Kim, S. C., Kim, J., Kim, C., Park, B., Park, S. S., Park, S. J., Park, E., Son, Y., Yoon, J., Lee, B., Lee, E., Lee, J. W., Lee, H. S., Joo, Y., Choi, J., Ha, T., Hwang, W., Hwang, I., Lee, J. Y., Oh, B., Lee, C. H., Lee, H. S., Kim, J. Y., Hwang, J. Y., Nam, S. H. \& Cho, M. (2013). J. Instrum. 8, P01019.

Siegbahn, H. \& Siegbahn, K. (1973). J. Electron Spectrosc. Relat. Phenom. 2, 319-325.

Siegbahn, K. (1969). ESCA Applied to Free Molecules. Amsterdam: North Holland. 
Soler, L., Casanovas, A., Ryan, J., Angurell, I., Escudero, C., PérezDieste, V. \& Llorca, J. (2019). ACS Catal. 9, 3641-3647.

Somorjai, G. A. (1978). Science, 201, 489-497.

Starr, D. E., Liu, Z., Hävecker, M., Knop-Gericke, A. \& Bluhm, H. (2013). Chem. Soc. Rev. 42, 5833-5857.

Tesch, M. F., Bonke, S. A., Jones, T. E., Shaker, M. N., Xiao, J., Skorupska, K., Mom, R., Melder, J., Kurz, P., Knop-Gericke, A., Schlögl, R., Hocking, R. K. \& Simonov, A. N. (2019). Angew. Chem. Int. Ed. 58, 3426-3432.

Timm, R., Head, A. R., Yngman, S., Knutsson, J. V., Hjort, M., McKibbin, S. R., Troian, A., Persson, O., Urpelainen, S., Knudsen, J., Schnadt, J. \& Mikkelsen, A. (2018). Nat. Commun. 9, 1412.
Usachov, D., Vilkov, O., Grüneis, A., Haberer, D., Fedorov, A., Adamchuk, V. K., Preobrajenski, A. B., Dudin, P., Barinov, A., Oehzelt, M., Laubschat, C. \& Vyalikh, D. V. (2011). Nano Lett. 11, 5401-5407.

Watanabe, M., Toyoshima, A., Azuma, Y., Hayaishi, T., Yan, Y. \& Yagishita, A. (2004). Proc. SPIE, 3150, 58-66.

Xu, H., Ma, L. \& Jin, Z. (2018). J. Energy Chem. 27, 146-160.

Yang, S. J., Choi, S., Odongo Ngome, F. O., Kim, K. J., Choi, S. Y. \& Kim, C. J. (2019). Nano Lett. 19, 3590-3596.

Yu, L., Takagi, Y., Nakamura, T., Sakata, T., Uruga, T., Tada, M., Iwasawa, Y., Masaoka, S. \& Yokoyama, T. (2019). J. Phys. Chem. C, 123, 603-611. 